\title{
Paired Associative Stimulation Enforces the Communication between Interconnected Areas
}

\author{
Domenica Veniero, ${ }^{1}$ Viviana Ponzo, ${ }^{1}$ and Giacomo Koch $^{1,2}$ \\ ${ }^{1}$ Non-Invasive Brain Stimulation Unit, Santa Lucia Foundation Istituto di Ricovero e Cura a Carattere Scientifico (IRCCS), 00179 Rome, Italy, and \\ ${ }^{2}$ Department of Neuroscience, University of Rome Tor Vergata, 00133 Rome, Italy
}

Paired associative stimulation (PAS) protocols induce forms of spike-timing-dependent-plasticity (STDP) when paired pulses are repeatedly applied with different timing over interconnected cortical areas such as the posterior parietal cortex (PPC) and the primary motor cortex (M1). However, the assessment of PAS effects is usually limited to M1 through recording of motor-evoked potential (MEP) amplitude. Here, by combining transcranial magnetic stimulation (TMS) with EEG we aimed at investigating PAS effects over both areas (PPC, M1) and the modulation induced on their connectivity in humans. In different PAS conditions, PPC preceded or followed M1 TMS by $5 \mathrm{~ms}$. We found that TMS-evoked potentials (TEPs) changed differently according to the long-term depression (LTD) or potentiation (LTP) after-effect assessed by MEPs, but did not vary at PPC level. Moreover, there was no change in local oscillatory power. However, there was a remarkable increase of coherence between the PPC and the M1 areas. When the PAS protocol induced LTD as revealed by MEPs, there was a specific increase of the coherence between PPC and M1 within the beta band. On the contrary, when PAS induced LTP, the coherence crucially increased in the alpha band. The same LTP results were confirmed when rotating the stimulating coil in M1 during the PAS protocol. In conclusion, we report new evidence that opposite STDP-like effects induced by corticocortical PAS are associated with increased communication between involved brain areas and that antithetic forms of STDP-like after-effects result in distinct cortical rhythms connectivity changes.

\section{Introduction}

Changes in synaptic strength represent a fundamental phenomenon through which learning and memory formation are achieved (Katz and Shatz, 1996). When a presynaptic cell fires few milliseconds before the postsynaptic cell, long-term potentiation (LTP) is produced, whereas the opposite temporal order results in a long-term depression (LTD), named spike-timingdependent-plasticity (STDP) (Sjöström et al., 2008). Recent studies have also formalized a location-dependency rule pointing to opposite timing requirements of distal inputs, named antihebbian STDP (Letzkus et al., 2006). As dendritic distance increase, a gradual shift of the timing requirements occurs, so that at apical dendrite the conventional rules are completely reversed. In line with these anti-hebbian rules, LTD is generated when presynaptic activation precedes the postsynaptic one and LTP during postsynaptic/presynaptic pairing (Froemke et al., 2010). Despite being widely accepted, little is known about these mechanisms in the intact brain, since most of the studies have been conducted in brain slices (Sjöström et al., 2008).

\footnotetext{
Received April 24, 2013; revised June 17, 2013; accepted July 18, 2013.

Author contributions: D.V. and G.K. designed research; D.V., V.P., and G.K. performed research; D.V. and G.K. analyzed data; D.V. and G.K. wrote the paper.

This work was supported by grants from the Italian Ministry of Health to G.K. (RF08.18, MR 08.7; GR 09.219). We are grateful to Dr. Silvia Picazio and Dr. Sonia Bonni for their help in data collection.

The authors declare no competing financial interests.

Correspondence should be addressed to Dr. Giacomo Koch, Laboratorio di Neurologia Clinica e Comportamentale, IRCCS Fondazione S. Lucia, Via Ardeatina 306, 00179 Rome, Italy. E-mail address: g.koch@hsantalucia.it.

DOI:10.1523/JNEUROSCI.1777-13.2013

Copyright $\odot 2013$ the authors $\quad 0270-6474 / 13 / 3313773-11 \$ 15.00 / 0$
}

In humans, hebbian-like mechanisms have been identified using transcranial magnetic stimulation (TMS). LTP- and LTDlike effects can be induced in the primary motor cortex (M1) following the repeated application of paired pulses over interconnected areas such as contralateral M1 (Rizzo et al., 2009), ventral premotor cortex (Buch et al., 2011), or supplementary motor area (Arai et al., 2011). Recently, by targeting the posterior parietal cortex (PPC) and M1, we provided evidence suggesting the existence of both hebbian and anti-hebbian phenomena in humans (Koch et al., 2013). As indexed by MEPs amplitude, the effects of repetitive PPC-M1 activation depended on the timing between the stimuli and, crucially, on the stimulation of specific neuronal populations (Koch et al., 2013). When PPC preceded $\mathrm{M} 1$, the paired associative stimulation protocol (PAS) led to a long-lasting decrease of M1 excitability (up to $20 \mathrm{~min}$ ) pointing to an LTD-like effect. When PPC followed M1stimulation, the same protocol induced a long-lasting increase of M1 excitability (up to $20 \mathrm{~min}$ ) indicating an LTP-like effect, satisfying the antihebbian STDP. However, when targeting different neuronal M1 populations by changing the induced current direction over M1 (Day et al., 1989; Ni et al., 2011), the induced STDP switched to classical hebbian rules (Koch et al., 2013).

This evidence came from the analysis of M1 excitability, since MEPs were used to verify local cortical plasticity. A crucial question is whether PAS effects are limited to M1 or affect the activity of the other targeted area. Moreover, the information about changes in connectivity between the two stimulated areas is completely lacking. Here, we used the combination of TMS with electroencephalography (EEG) to directly address these issues. TMS/ 
A

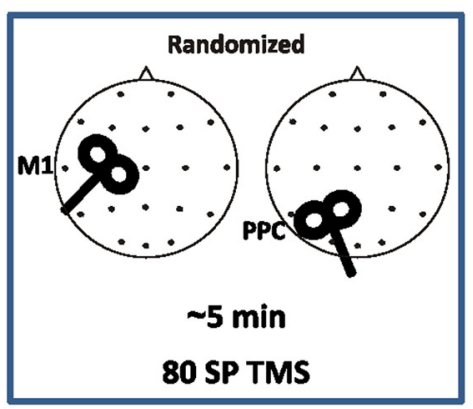

Pre-PAS

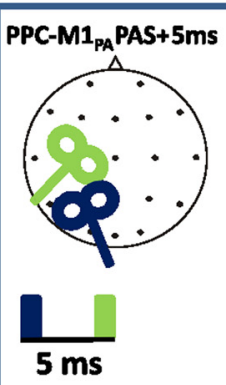

PAS stimulation

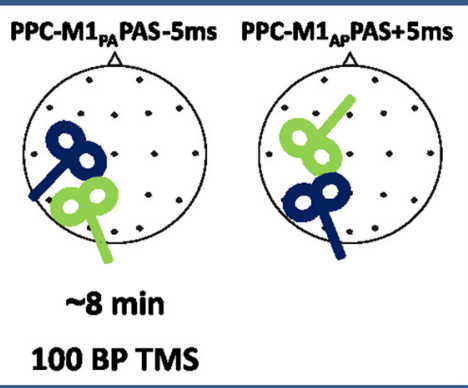

Post-PAS

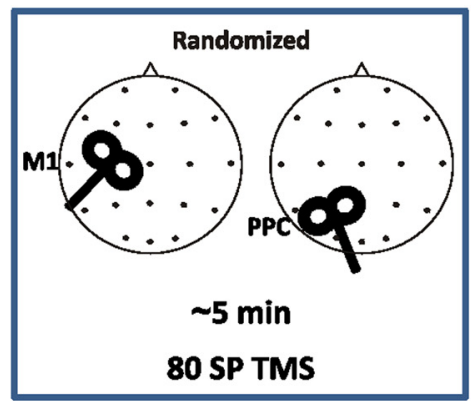

B
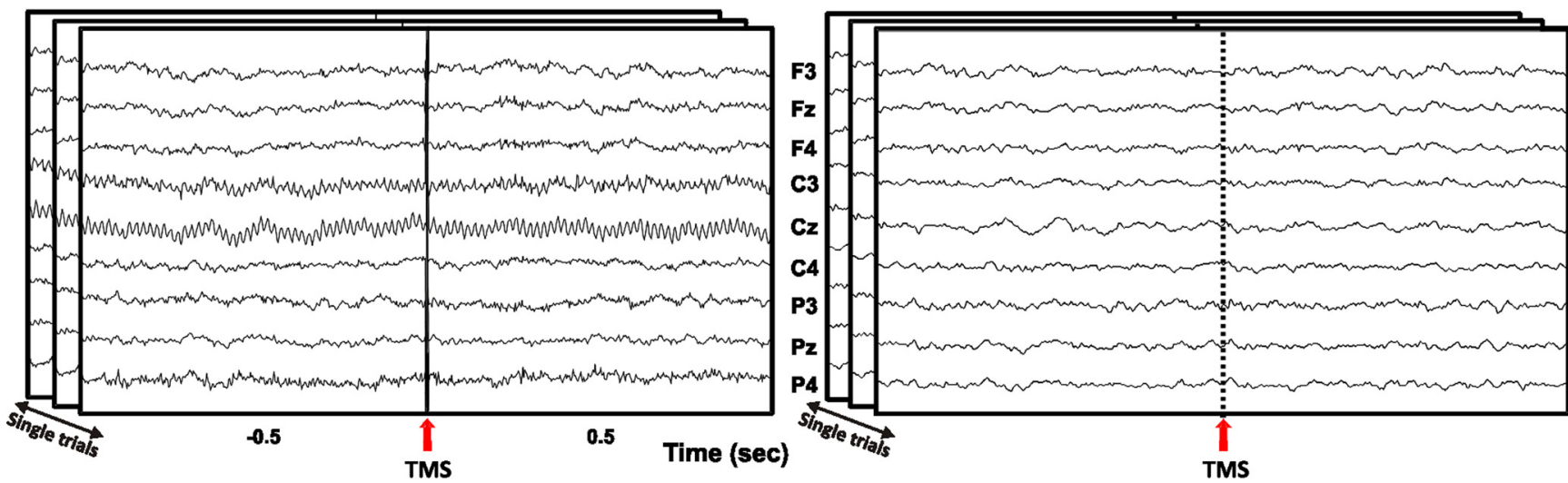

Figure 1. Schematic representation of the experimental procedure. $A$, Eighty single pulse TMS were delivered before and after the administration of three different PAS protocols in a randomized order over M1 or PPC of the left dominant hemisphere. For all experiments the interstimulus interval was at $5 \mathrm{~ms}$. The order of pulse delivery was changed across condition, so that positive paring indicate that the PPC input precedes the M1 pulse while negative paring indicate that the PPC input is applied after the M1 pulse. The orientation of the stimulating coil over M1 was also changed from posterior-anterior $\left(M 1_{\mathrm{PA}}\right)$ to anterior-posterior $\left(\mathrm{M1}_{\mathrm{AP}}\right)$. The blue rectangle and coil indicate the first delivered pulse. $\boldsymbol{B}$, Data processing. An epoch of $1 \mathrm{~s}$ of unprocessed data is shown before (left) and after artifacts removal (right) from one representative subject for nine electrodes. Data refers to the stimulation of M1.

EEG allows to measure physiological signals generated at the cortical level by TMS, including those generated in the so-called silent-areas such as PPC (Miniussi and Thut, 2010) and to assess modifications of cortical connectivity (Ilmoniemi et al., 1997). First, the study of TMS-evoked potentials (TEPs) was performed to investigate the modulation induced on M1 and PPC excitability. In a second step, TMS-induced oscillations and connectivity changes were investigated.

\section{Materials and Methods}

\section{Subjects}

Thirteen right-handed subjects $(7$ female; mean age $=27.6 \pm 3.2$; range $=24-32$ years) participated in the study after giving their written informed consent. None of the participants had any contraindication to TMS or any neurological, psychiatric, or other relevant medical problems (Rossi et al., 2009). The protocol was performed in accordance with ethical standards and was approved by the local Ethical Committee.

\section{General procedure}

Subjects were seated in a comfortable armchair in a dimly illuminated, electrically shielded, and sound-proof room with their hands pronated in a relaxed position. During the experiments, subjects were required to focus on a central fixation point.

As depicted in Figure 1A, bifocal TMS was applied to repeatedly activate the connection between the PPC and the M1 of the left dominant hemisphere (Koch et al., 2007, 2008) with the left PPC TMS preceding $\left(\mathrm{PPC}-\mathrm{M}_{\mathrm{PA}} \mathrm{PAS}-5 \mathrm{~ms}\right)$ or following $\left(\mathrm{PPC}-\mathrm{Ml}_{\mathrm{PA}}\right.$ PAS $-5 \mathrm{~ms}$ ) the $\mathrm{M} 1$ stimulation by $5 \mathrm{~ms}$. All subjects underwent these two PAS conditions on two different days in a randomized order. According to our previous results (Koch et al., 2013), a third experimental condition was performed on a subsample of eight subjects to test whether PAS effects at cortical level could vary depending on the preferential activation of different neuronal populations by varying the direction of the induced current over M1 during PAS delivery. Indeed, it is known that the neuronal populations activated by TMS in M1 depend on the direction of the induced current in the brain (Day et al., 1989; Di Lazzaro et al., 2006). To this end, during the PAS procedure, the induced current direction over $\mathrm{M} 1$ was changed from posteroanterior (PA) to anteroposterior (AP) and the interstimulus interval (ISI) was set at $+5 \mathrm{~ms}$ (PPC-M1 ${ }_{\mathrm{AP}} \mathrm{PAS}-5 \mathrm{~ms}$ ) (Fig. 1A). To evaluate cortical effects of bifocal stimulation for all conditions $\left(\mathrm{PPC}-\mathrm{M} 1_{\mathrm{PA}} \mathrm{PAS}-5 \mathrm{~ms}\right.$, PPC-M1 $1_{\mathrm{PA}} \mathrm{PAS}-5 \mathrm{~ms}$, and $\mathrm{PPC}-\mathrm{M} 1_{\mathrm{AP}}$ $\mathrm{PAS}-5 \mathrm{~ms}), 80$ single pulse TMS were applied in randomized order to $\mathrm{M} 1$ and PPC before and after PAS while continuously acquiring EEG.

\section{TMS}

During PPC-M $1_{\mathrm{PA}}$ PAS $-5 \mathrm{~ms}$ and PPC-M1 $1_{\mathrm{PA}}$ PAS $-5 \mathrm{~ms}$, PAS stimulation was applied by means of two high-power Magstim 200 machines (Magstim Co.), delivering single monophasic current pulses. MEPs were recorded using $9 \mathrm{~mm}$ diameter, $\mathrm{Ag}-\mathrm{AgCl}$ surface cup electrodes. The active electrode was placed over the belly muscle, while the reference electrode was located over the metacarpophalangeal joint of the index finger. Responses were amplified using a Digitimer D360 amplifier (Digitimer) through filters set at $20 \mathrm{~Hz}$ and $2 \mathrm{kHz}$ with a sampling rate of 5 $\mathrm{kHz}$, then recorded by a computer using SIGNAL software (Cambridge Electronic Devices). For M1 TMS, the coil was positioned over the hand motor area of left M1, defined as the point where stimulation evoked the largest MEPs from the contralateral FDI muscle. The stimulator for M1 was connected to a small custom-made figure-of-eight-shaped coil (external diameter $50 \mathrm{~mm}$ ). The intensity of M1 TMS was adjusted to evoke a MEP of $\sim 1 \mathrm{mV}$ peak-to-peak in the relaxed contralateral FDI muscle. The coil was positioned to induce a posterior-to-anterior (PA) directed 
current, being held posterolaterally at an angle of $\sim 45^{\circ}$ to the midline. To best activate the ipsilateral PPC-M1 connection, the conditioning stimulus was applied over the left PPC at an intensity of $90 \%$ of the ipsilateral resting motor threshold (RMT). RMT was defined as the lowest intensity that evoked five small responses $(\sim 50 \mu \mathrm{V})$ in the contralateral FDI muscle, in a series of 10 stimuli when the subject kept the FDI muscles relaxed in both hands (Rossini et al., 1994). The magnetic pulse over PPC was applied using a second small custom-made figure-of-eight-shaped coil (external diameter, $50 \mathrm{~mm}$ ). To precisely position the coil over the PPC sites, a neuronavigation system (Softaxic, E.M.S.) was used, using individual T1-weighted magnetic resonance imaging volumes as anatomical reference [for a detailed description, see the study by Koch et al. (2010)]. To target PPC, the coil was positioned over the angular gyrus, close to a posterior part of the adjoining caudal intraparietal sulcus (cIPS). The coordinates of the left PPC were stored and used for neuronavigation to ensure comparable stimulation conditions across sessions (mean normalized MNI coordinates of left PPC site were as follows: $-46.8 \pm 5.8$, $-67.1 \pm 4.9$, and $42.6 \pm 6.1 \mathrm{~mm}$ ). The center of the coil was positioned tangentially to the skull with the handle pointing downward and rotated medially by $15^{\circ}$ (Koch et al., 2010, 2011). The PAS protocol consisted of 100 pairs of stimuli continuously delivered at a rate of $0.2 \mathrm{~Hz}$ for $\sim 8.3$ min. The left PPC TMS preceded (PPC-M1 $1_{\mathrm{PA}} \mathrm{PAS}-5 \mathrm{~ms}$ ) or followed (PPC-M1 $1_{\mathrm{PA}} \mathrm{PAS}-5 \mathrm{~ms}$ ) the M1 stimulation by $5 \mathrm{~ms}$.

During PPC-M $1_{\mathrm{AP}}$ PAS $-5 \mathrm{~ms}$, all parameters were kept identical to the other conditions, but PAS was performed so that the induced current direction over M1 changed from PA to AP. To this end, $\mathrm{M} 1_{\mathrm{AP}}$ TMS was applied over the hot spot of the FDI by rotating the coil handle of $180^{\circ}$ from the PA direction. The intensity of $\mathrm{M}_{\mathrm{AP}}$ stimulation was adjusted to obtain peak-to-peak MEP amplitude of $\sim 1 \mathrm{mV}$. In this session, PPC TMS always preceded M1 stimulation by $5 \mathrm{~ms}$ (PPC-M1 $\left.1_{\mathrm{AP}} \mathrm{PAS}-5 \mathrm{~ms}\right)$. To evaluate the effect of paired stimulation for all conditions $\left(\mathrm{PPC}-\mathrm{M} 1_{\mathrm{PA}}\right.$ PAS $-5 \mathrm{~ms}, \mathrm{PPC}-\mathrm{M}_{\mathrm{PA}} \mathrm{PAS}-5 \mathrm{~ms}$, and PPC-M1 $1_{\mathrm{AP}}$ PAS $\left.-5 \mathrm{~ms}\right), 80$ single pulse TMS were applied in randomized order to M1 and PPC before and after PAS while continuously acquiring EEG. Each experimental session was performed in different days, at least 1 week apart in a randomized order.

\section{EEG/TMS recordings}

TMS-compatible EEG equipment (BrainAmp 32MRplus, BrainProducts) was used for recording EEG activity from the scalp. The EEG was continuously acquired from 20 scalp sites positioned according to the 10-20 International System, using electrodes mounted on an elastic cap. Additional electrodes were used as ground and reference. The ground electrode was positioned in $\mathrm{AFz}$, while an active reference was positioned on the tip of the nose. The signal was bandpass filtered at $0.1-1000 \mathrm{~Hz}$ and digitized at a sampling rate of $5 \mathrm{kHz}$. To minimize overheating of the electrodes proximal to the stimulating coil, TMS-compatible $\mathrm{Ag} / \mathrm{AgCl}$ sintered ring electrodes were used. Skin/electrode impedance was maintained $<5 \mathrm{k} \Omega$. Horizontal EOG (HEOG) was recorded from electrodes positioned on the outer canthi of both eyes, and vertical EOG (VEOG) from electrodes located beneath the right eye recorded vertical eye movements and blinks. To reduce auditory contamination of EEG induced by coil clicks, subjects wore earplugs throughout the experiment and were required to focus on a central fixation point. The TMS intensity was set to evoke a MEP of $\sim 1 \mathrm{mV}$ peak-to-peak in the relaxed contralateral FDI muscle regardless of the stimulated area (M1 or PPC).

\section{MEP analysis}

When TMS was performed over M1, MEPs were collected concurrently to EEG recordings. MEP amplitude was analyzed to investigate the effects of each PAS protocol over corticospinal excitability and to further replicate our previous results (Koch et al., 2013). Mean peak-to-peak MEPs amplitude evoked before and after each PAS protocol was compared by means of paired $t$ tests.

\section{EEG analysis}

To characterize how PAS affects cortical dynamics, EEG data were analyzed off-line to recordings using BrainVision Analyzer2 (BrainProducts) and EEGLAB (Delorme and Makeig, 2004) implemented in Matlab 7.8 (The MathWorks).
Time-domain analysis: TEPs

EEG signals were first rereferenced to the average of all electrodes and high-pass filtered at $0.1 \mathrm{~Hz}$ (butterworth zero phase filter). For each subject, condition, and target area (M1 or PPC stimulation, before and after each PAS administration), the epoching of EEG responses started $100 \mathrm{~ms}$ before and ended $600 \mathrm{~ms}$ after the TMS pulse. All epochs were baseline corrected to a time period of $100 \mathrm{~ms}(-100-0 \mathrm{~ms})$ recorded before TMS delivery. Epochs with excessively noisy EEG, eye-movement artifacts (blinks or saccades) or muscle artifacts were excluded from further analysis by visual inspection. Because of a low quality of the EEG recording, due to exceeding artifact contaminations, one subject had to be excluded from the study before postprocessing and was not considered for frequency domain analysis (see below). Independent component analysis (ICA) was then used to identify and remove components reflecting residual muscle activity, eye movements, blink-related activity, and residual TMS-related artifacts. In particular, ICs were removed according to three criteria: a topography indicating the activation of left temporalis muscle (TM), characterized by a peak over the electrodes close to TM muscle, such as F7, and by an activation of opposed polarity mainly recorded over frontocentral electrodes, such as C3 (Mutanen et al., 2013); the ICs occurred within 15-20 ms from the TMS pulse; the amplitude of the ICs exceeded $50 \mu \mathrm{V}$. No more than four components were removed in each subject.

After these steps, the artifact induced by pulse delivery, typically lasting 2-6 ms with our equipment (Veniero et al., 2009), was removed using cubic interpolation for a conservative $10 \mathrm{~ms}$ interval following the TMS pulse (Thut et al., 2011) (Fig. 1B). Total EEG activity was assessed using the global mean field power (GMFP), calculated as follows:

$$
\operatorname{GMFP}(t)=\sqrt{\frac{\left(\sum_{i}^{k}\left(V i(t)-V_{\text {mean }}(t)\right)^{\wedge} 2\right\rfloor}{K}},
$$

where $t$ is time, $K$ the number of channels, $V_{\mathrm{i}}$ the voltage in channel $i$ averaged across subjects, and $V_{\text {mean }}$ is the mean of the voltages in all channels.

To investigate whether the three PAS protocols were able to modulate cortical excitability, for each subject the amplitudes of the first four peaks occurring within the $300 \mathrm{~ms}$ following the TMS pulse in the GMFP in the two test phases were identified. ANOVA for repeated measures was performed on the peaks observed in the GMFP with the factors Time (before or after PAS) and Peak. The Huynh-Feldt $\varepsilon$ correction factor was applied where appropriate to compensate for the possible effects of nonsphericity in the compared measurements. Post hoc tests were performed to investigate significant effects, by means of planned $t$ test as appropriate for multiple comparisons.

\section{Frequency-domain analysis}

Oscillatory activity. Preprocessing epochs were of $2 \mathrm{~s}$ duration $(-1 \mathrm{~s}$ to $+1 \mathrm{~s}$ from TMS pulse onset). Single trials were visually inspected to exclude epochs with excessively noisy EEG, eye movement artifacts, or muscle artifacts. As for time-domain analysis, the first $10 \mathrm{~ms}$ following each TMS pulse were interpolated. ICA was then used to identify and remove components reflecting residual artifactual activity. To analyze the oscillatory activity induced by the TMS pulse, we calculated the timefrequency responses for all epochs, spanning from $1000 \mathrm{~ms}$ before to $1000 \mathrm{~ms}$ after pulse onset. A complex Morlet wavelet $(2-40 \mathrm{~Hz}, 20$ frequency steps, $c=5$ ) was applied to each single epoch in each subject, normalizing the data to a pre-TMS period of $500 \mathrm{~ms}(-500-0 \mathrm{~ms})$. After Wavelet computations, data were averaged. To evaluate the changes in the induced activity related to distinct PAS protocols, for each condition and stimulated area, we extracted the frequency range between 4 and 7 $\mathrm{Hz}$ (theta), 8-12 Hz (alpha), and $13-30 \mathrm{~Hz}$ (beta) from the wavelet dataset. Induced activity before and after each PAS condition and stimulated cortex were compared by means of separated ANOVAs with factors Time (before PAS vs after PAS) and Electrode. The normal distribution was tested using the Kolmogorov-Smirnov test (for all $p>$ 0.2 ). The Huynh-Feldt $\varepsilon$ correction factor was applied where appropriate to compensate for the possible effects of nonsphericity in the compared measurements. Post hoc tests were performed to investigate 


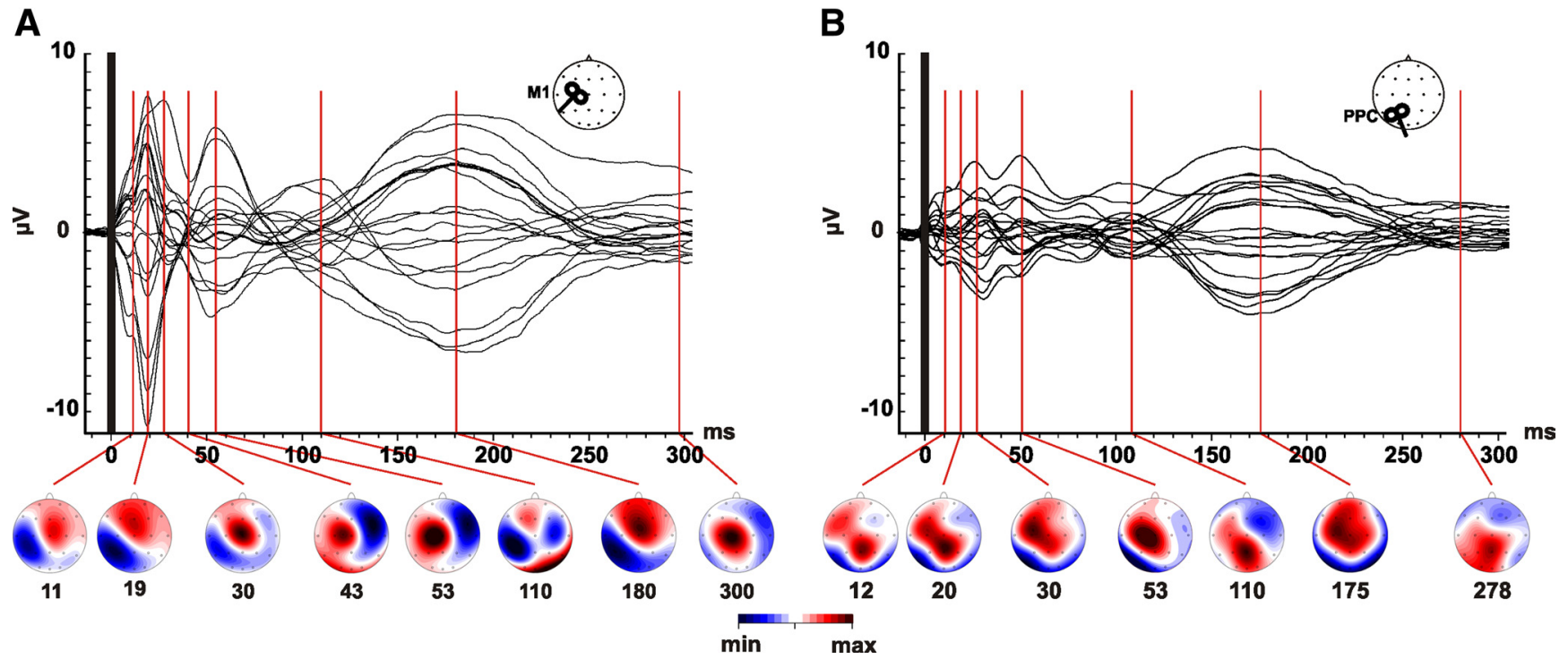

Figure 2. Spatiotemporal dynamics of TEPs. Grand-average of TEPs recorded at all electrodes, superimposed in a butterfly plot as a result of M1 $(\boldsymbol{A})$ and PPC $(\boldsymbol{B})$ stimulation. The time of pulse delivery is indicated by a vertical black bar. Data are obtained by collapsing two pre-PAS conditions (PPC-M1 $\left.1_{\mathrm{PA}}+5 \mathrm{~ms}, \mathrm{PPC}-\mathrm{M} 1_{\mathrm{PA}}-5 \mathrm{~ms}\right)$. In the lower part of each panel, topographic maps are displayed at specific time points corresponding to the TEP latencies as reported in the Results. Map voltage is color coded according to the minimum (blue) and maximum (red) voltage ( $\mu \mathrm{V}$ ) of each response.

significant effects, by means of planned $t$ test as appropriate for multiple comparisons.

Interregional functional connectivity. Analyses of EEG signals were computed for two epochs: a reference period (from $501 \mathrm{~ms}$ to $1 \mathrm{~ms}$ before each pulse delivery) and interest period (from 0 to $500 \mathrm{~ms}$ after each pulse delivery). For each subject and condition, the power spectra were estimated for each single epoch and all frequency bins between 0 and $100 \mathrm{~Hz}$ by means of a Fast Fourier transform (Hamming window; frequency resolution $1.5 \mathrm{~Hz}$ ). Accordingly, coherence values were calculated for all frequency bins following the following formula:

$$
\operatorname{Coh}_{c_{1}, c_{2}}(f)=\frac{\left|\operatorname{CS}\left(c_{1}, c_{2}\right)(f)\right|^{2}}{\left(\left|\operatorname{CS}\left(c_{1}, c_{1}\right)(f) \| \operatorname{CS}\left(c_{2}, c_{2}\right)(f)\right|\right)},
$$

with CS $=\left(c_{1}, c_{2}\right)(f)=\operatorname{Sigma} ; c_{1}, i(f) c_{2}, i(f)^{*}$, where $i$ represents the epoch number. This formula extends the Pearson's correlation coefficient to complex number pairs. Accordingly, the coherence spectrum of two signals $\left(c_{1}, c_{2}\right)$ is calculated as normalization of cross-spectrum by the two auto-spectra. For each frequency $f$, the coherence value is a real number between 0 and 1 . Broad-band coherence values were then obtained by averaging the coherence values over all epochs for the theta $(4-7 \mathrm{~Hz})$, alpha $(8-12 \mathrm{~Hz})$, and beta $(13-30 \mathrm{~Hz})$ bands. When testing for changes in M1 connectivity, the calculation was performed for a combination of the C3 electrode (the nearest channel to the individual M1 "hot-spot") with all other channels. When testing for changes in left PPC connectivity, a combination of P3 electrode (the nearest electrode to individual PPC hot-spot) with all signals recorded from each recording site was instead considered. Finally, event-related coherence $\left(\mathrm{ERCoh}_{\mathrm{C} 1 \mathrm{C} 2}\right)$ was obtained by subtracting the reference period value ( Coh $_{\mathrm{ClC} 2}$ Reference) from the corresponding interest period value (Coh $_{\mathrm{C} 1 \mathrm{C} 2}$ Interest), according to the following formula:

$$
\mathrm{ERCoh}_{\mathrm{clc2}}=\mathrm{Coh}_{\mathrm{c1c2}} \text { Interest }-\mathrm{Coh}_{\mathrm{clc2}} \text { Reference. }
$$

Therefore, a coherence increase in the frequency band during the period of interest $\left(\mathrm{Coh}_{\mathrm{C} 1 \mathrm{C} 2}\right.$ Interest) relative to the reference period $\left(\mathrm{Coh}_{\mathrm{C} 1 \mathrm{C} 2}\right.$ Reference) is expressed as a positive value, while a coherence magnitude decrease is expressed as a negative value (Pfurtscheller and Lopes da Silva, 1999; Fuggetta et al., 2008).

A separate ANOVA with repeated measures was performed for each frequency band of interest (theta, alpha, beta), stimulated cortex (M1, $\mathrm{PPC}$ ), and condition (PPC-M1 $1_{\mathrm{PA}} \mathrm{PAS}+5$ or -5 and $\mathrm{PPC}-\mathrm{M} 1_{\mathrm{AP}} \mathrm{PAS}-5$ $\mathrm{ms}$ ) testing the factors Time (before vs after PAS administration) and
Pair (C3- or P3- paired with all channels, 19 couples). The Huynh-Feldt correction factor was applied to compensate for nonsphericity in the compared measurements. Post hoc tests were performed to investigate significant effects, by means of planned $t$ tests.

Finally, to verify whether there was any correspondence between the modulatory effects of PAS protocols on TEP amplitude and the modulatory effects on the ERcoh, a Pearson's correlation coefficient $(p<0.05)$ was calculated between the changes of significantly modulated peak of the GMFP and the changes in ERcoh of a C3-P3 pair. The correlation was calculated on the data recorded during M1 single pulse stimulation.

\section{Results}

Time-domain analysis: TEPs

As expected, TMS evoked an EEG activity lasting up to $300 \mathrm{~ms}$ regardless of the stimulated cortex, resulting in a sequence of deflections of alternating positive and negative polarity starting a few milliseconds after stimulation (Komssi et al., 2002; Bonato et al., 2006; Veniero et al., 2010). Figure 2 illustrates the distribution of the TEPs components at all recording sites evoked by M1 $(A)$ and PPC $(B)$ stimulation. When TMS was performed over M1 (Fig. 2A), the earliest pattern $(11 \pm 1 \mathrm{~ms})$ was a dipole centered over the side of stimulation with a positivity peaking over $\mathrm{Cz}$ and an ipsilateral negativity involving parietal and left-central sites. It is noteworthy that, since the latency of this early component is closed to the interpolated interval $(0-10 \mathrm{~ms})$, we decided to exclude this component from statistical analysis. This dipolar pattern reached its maximal amplitude when at $18 \mathrm{~ms}$ (18 $\pm 2 \mathrm{~ms})$. This distribution evolved into a widespread positivity centered over $\mathrm{Cz} \sim 30 \mathrm{~ms}$ after TMS ( $29 \pm 3 \mathrm{~ms}$ ), indicating the spreading of activation from the stimulated cortex toward the contralateral hemisphere (Komssi et al., 2002; Veniero et al., 2010). Indeed, after $40 \mathrm{~ms}$ ( $39 \pm 5 \mathrm{~ms}$ ) and $50 \mathrm{~ms}(50 \pm 6 \mathrm{~ms})$, a strong negativity peaked at left central (C4) and frontal (F4) together with a positivity centered over $\mathrm{Cz}$ and $\mathrm{C} 3$. The TEP occurring at $110 \mathrm{~ms}$ $(100 \pm 5 \mathrm{~ms})$ was represented by a large negativity peaking over centroparietal sites of the stimulated hemisphere and a concurrent less huge but still evident negativity over centrofrontal right sites. Finally, two components peaked at $180(180 \pm 4 \mathrm{~ms})$ and $300 \mathrm{~ms}(300 \pm 10 \mathrm{~ms})$, the first showing a topographical distri- 
A

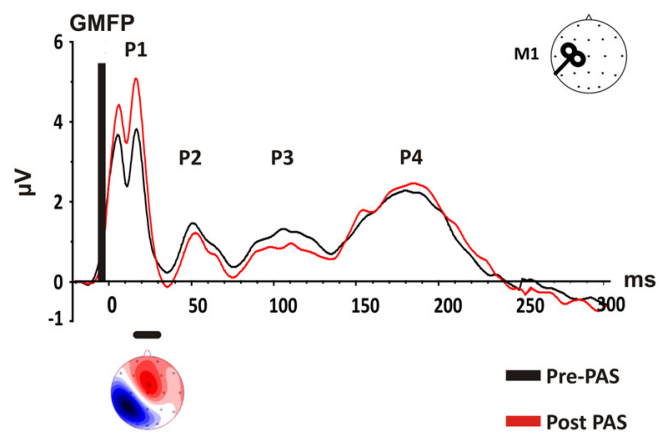

B

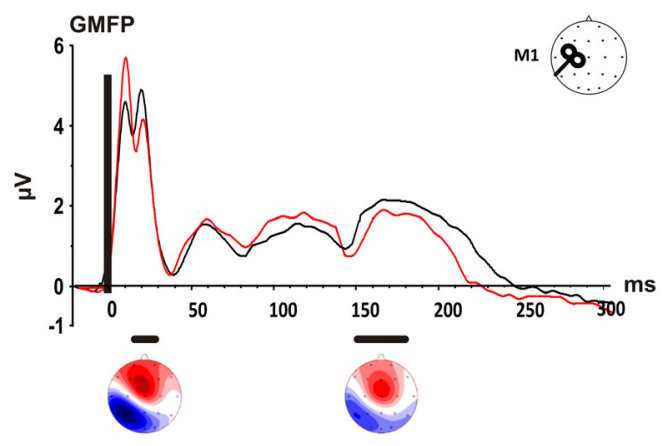

C

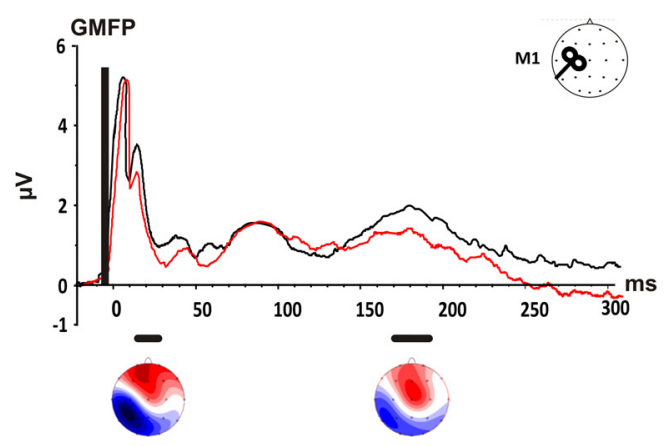

PPC-M1PA +5ms
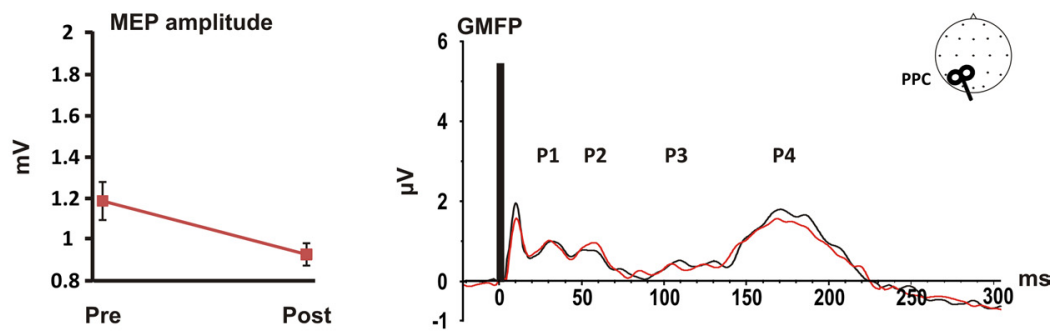

PPC-M1PA -5ms
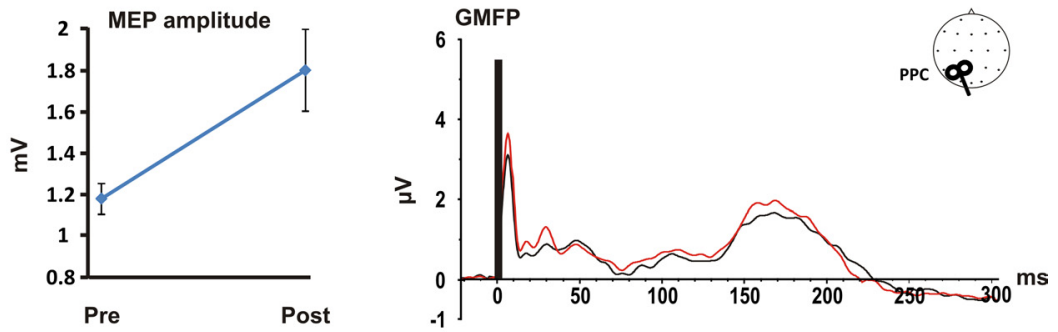

PPC-M1AP +5ms

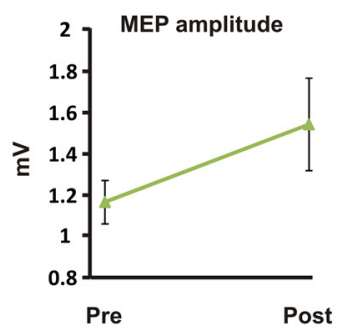

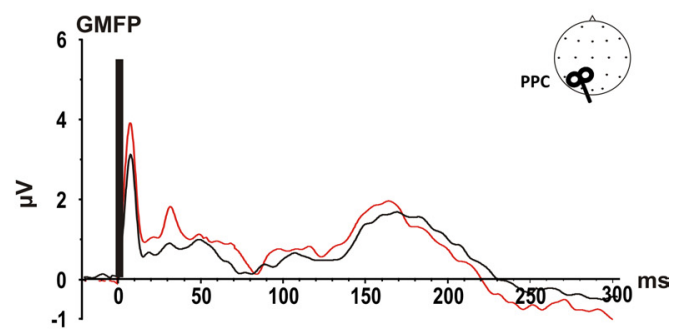

Figure 3. Global cortical reactivity changes induced by PAS protocols. For each PAS condition, the GMFP is shown for M1 (left) and PPC (right) stimulation. The central panels indicate the effects of each protocol over the MEP amplitude. The bottom horizontal black lines indicate significant differences between pre-PAS and post-PAS testing. For each significantly modulated peak, the topographic map is also shown. $A, P P C-M 1_{P A}+5 \mathrm{~ms}$. When PPC repeatedly preceded $\mathrm{M} 1$ stimulation by $5 \mathrm{~ms}$, single pulse TMS over M1 revealed a significant increase of P1 accompanied with a significant reduction of MEPs amplitude, whereas no changes was detected for PPC testing. B, PPC-M1 $1_{P A}-5 \mathrm{~ms}$. When PPC repeatedly followed M1 stimulation by $5 \mathrm{~ms}$, single pulse TMS over M1 revealed a significant decrease of $\mathrm{P} 1$ and $\mathrm{P} 4$ accompanied with a significant $\mathrm{MEP}$ amplitude increment, whereas no changes was detected for PPC testing. C, PPC-M1 $1_{A P}+5 \mathrm{~ms}$. When PPC repeatedly preceded $\mathrm{M1}_{\mathrm{AP}}$ stimulation by $5 \mathrm{~ms}$, a significant decrease of amplitude for $\mathrm{P} 1$ and $\mathrm{P} 4$ after TMS pulse over $\mathrm{M} 1$ was accompanied with a significant MEP amplitude increment, whereas no changes was detected for PPC testing.

bution comparable to the early latency components (11 and 18 $\mathrm{ms}$ ) and the latter peaking over C3 and Cz.

When the stimulation was performed over PPC, TEPs were characterized by a smaller amplitude (Fig. $2 B$ ) and by a less pronounced propagation of activation from the hot-spot toward connected areas. As for M1 stimulation, the first component $(12 \pm 3 \mathrm{~ms}$ ) formed a dipolar field centered over P3. After $20 \mathrm{~ms}$ (18 $\pm 3 \mathrm{~ms}$ ) from the TMS pulse, the scalp distribution moved toward more anterior sites with a huge positivity recorded from $\mathrm{Pz}$ and C3. Afterward, the central positivity lateralized to the side of stimulation ( $30 \pm 2 \mathrm{~ms}$ ) evolved into a widespread positivity with a maximum over the central electrodes $(53 \pm 2 \mathrm{~ms})$. At 110 $\mathrm{ms}(110 \pm 4 \mathrm{~ms})$, a large positivity over $\mathrm{Pz}$ was associated with a negativity recorded from the centrofrontal electrodes of nonstimulated hemisphere. The latter components ( $175 \pm 5 \mathrm{~ms}$ and $279 \pm 8 \mathrm{~ms}$ ) were again characterized by a central activation followed by a low-amplitude TEP forming a dipole centered over central regions.

To evaluate the effects of distinct PAS protocols over cortical reactivity, the total activation evoked by TMS was calculated by means of the GMFP before and after each PAS condition. Figure 3 shows GMFP results when M1 or PPC excitability changes were assessed. As it can be seen, four peaks were identified for M1 TMS and PPC TMS (P1, P2, P3, P4). The peaks had similar latencies when compared between the pre-PAS and post-PAS test phases and were also comparable within the same area across conditions. 
A

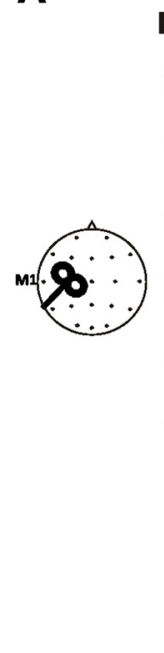

C3

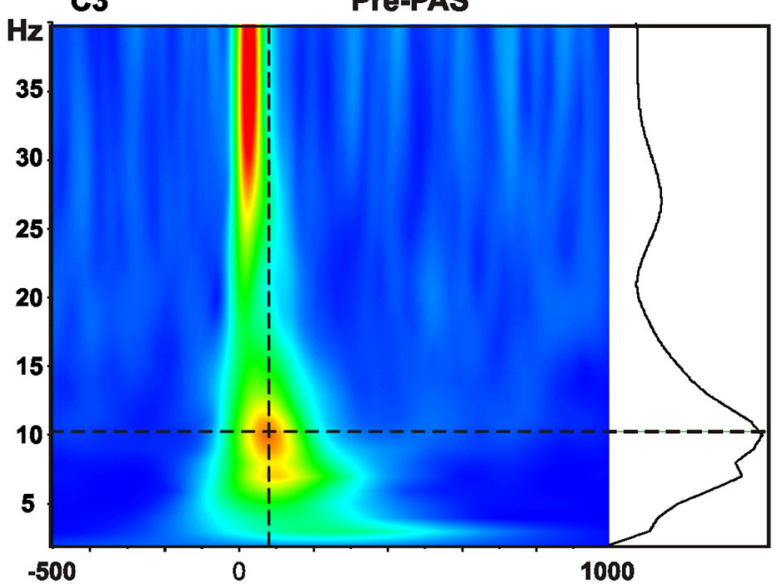

Pre-PAS

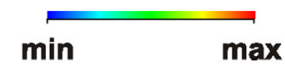

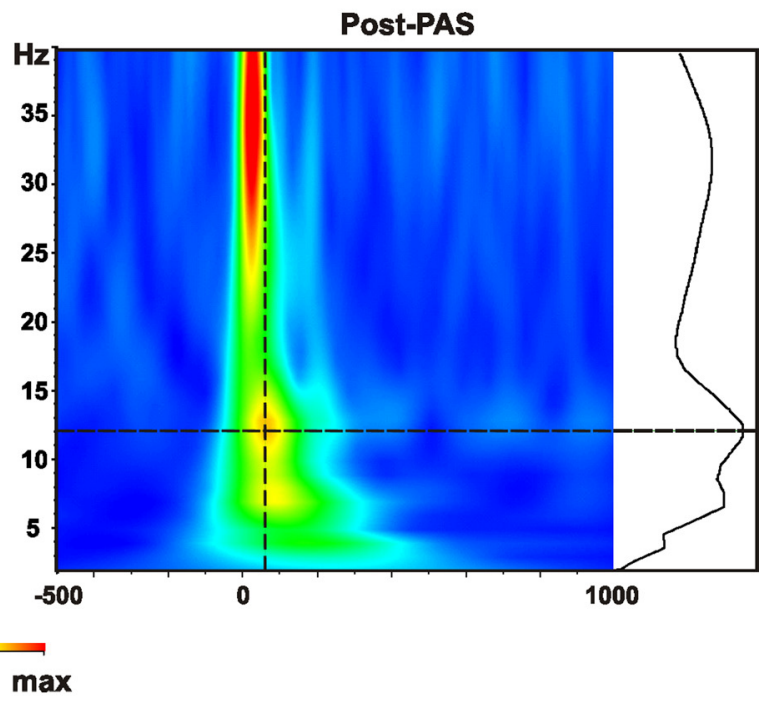

B

P3

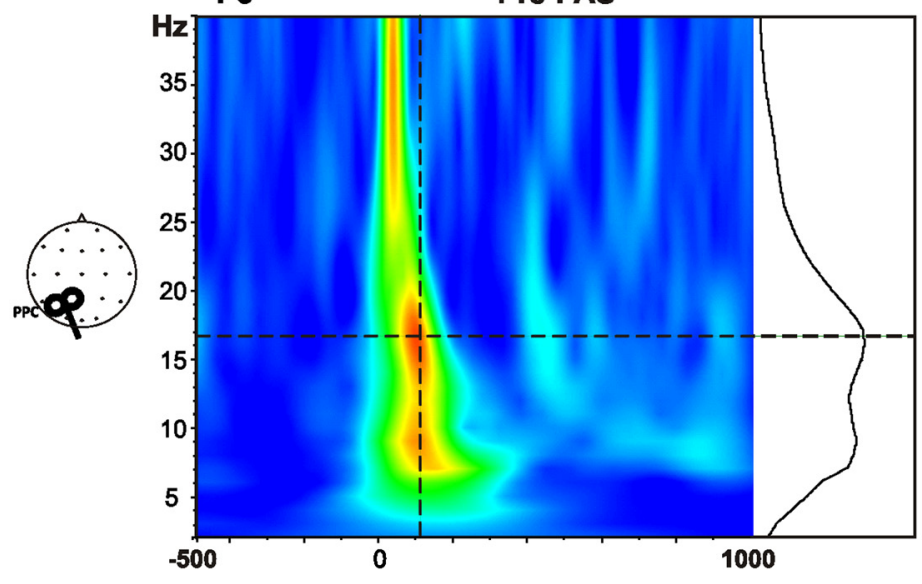

Pre-PAS

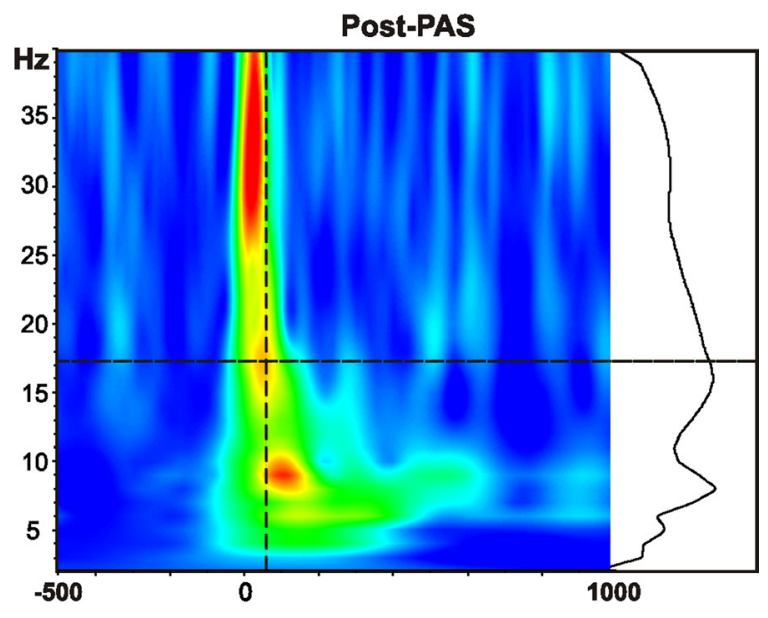

Figure 4. TMS-induced activity. Grand-averaged time-frequency plots are depicted for a representative condition (PPC-M1PA $+5 \mathrm{~ms})$ depicted for C3 ( $A$ ), representing the closest electrode to M1 hot-spot and P3 $(\boldsymbol{B})$, the closest recording site to PPC, before and after PAS administration. The black graph plotted at the right of each time-frequency plot illustrates the power spectrum profile induced by the TMS pulse at the intersection of the two dotted green lines, indicating the maximum induced power.

When single pulse TMS was applied to M1, the evoked peaks occurred with a latency of $18 \pm 2,53 \pm 4 ; 108 \pm 5 ; 186 \pm 2 \mathrm{~ms}$. ANOVA performed for the PPC-M1 $1_{\mathrm{PA}}+5 \mathrm{~ms}$ condition revealed a significant Time $\times$ Peak interaction $\left(F_{(3,33)}=3.66 ; p=0.022\right)$, due to a significant increase of $\mathrm{P} 1$ amplitude after PAS administration $(p=0.004$; Fig. $3 A)$. For PPC-M1 $1_{\mathrm{PA}}-5 \mathrm{~ms}$ condition a significant Time $\times$ Peak interaction again emerged $\left(F_{(3,33)}=\right.$ $3.27 ; p=0.032)$. However, this condition lead to a decrease in $\mathrm{P} 1$ $(p=0.009)$ and $\mathrm{P} 4(p=0.019)$ amplitude (Fig. $3 B)$. Similarly, the ANOVA performed for PPC-M $1_{\mathrm{AP}}+5 \mathrm{~ms}$ condition indicated that P1 and P4 amplitude were decreased after PAS administration as indexed by a significant Time $\times$ Peak interaction $\left(F_{(3,21)}=3.62 ; p=0.029\right)$ and post hoc tests $(\mathrm{P} 1: p=0.05 ; \mathrm{P} 4: p=$ 0.001 ) (Fig. $3 C$ ). Interestingly, the topographical distribution associated with the significantly modulated peaks in all PAS protocols was characterized by a dipole centered over the stimulated area, that is, M1 (C3). As shown in Figure 3 when the GMFP was calculated for the PPC stimulation, the peaks occurred with a latency of $28 \pm 3,50 \pm 4,109 \pm 6,160 \pm 9 \mathrm{~ms}$, but ANOVA revealed no significant modulation (all $p>0.05$ ), indicating that regardless of the applied protocols, PAS was unable to induce changes in parietal cortex excitability.

\section{MEP modulations}

Clear modulation of MEPs amplitude was present as can be seen in Figure 3 (middle part of each panel) after all PAS conditions. In particular, in agreement with our previous data (Koch et al., 2013), PPC-M1 $1_{\text {PA }}$ PAS $+5 \mathrm{~ms}$ resulted in a decrease of MEPs amplitude $(t=2.32 ; p=0.04)$, whereas $\mathrm{PPC}-\mathrm{M} 1_{\mathrm{PA}} \mathrm{PAS}-5 \mathrm{~ms}$ caused a significant increase in MEP amplitude $(t=3.92 ; p=$ $0.002)$, thus satisfying the anti-hebbian STDP rules. However, when the current direction induced over M1 was reversed to AP direction, an increased corticospinal excitability was found as revealed by pre-MEP and post-MEP amplitude comparison for $\mathrm{PPC}-\mathrm{M} 1_{\mathrm{AP}} \mathrm{PAS}-5 \mathrm{~ms}(t=2.46 ; p=0.043)$, according to classical hebbian STDP.

\section{Oscillatory activity}

Figure 4 shows the results of wavelet analysis performed before and after a representative PAS condition ( $\mathrm{PPC}-\mathrm{M} 1_{\mathrm{AP}} \mathrm{PAS}-5 \mathrm{~ms}$ ) when the single pulses where delivered over M1 (Fig. 4A) and PPC (Fig. 4B). As expected, the stimulation performed over M1 consistently induced an oscillatory activity in the alpha range (Fig. 4A), whereas the PPC stimulation mainly induced a beta band activity (Fig. $4 B$ ) in both testing phase (Rosanova et al., 
A

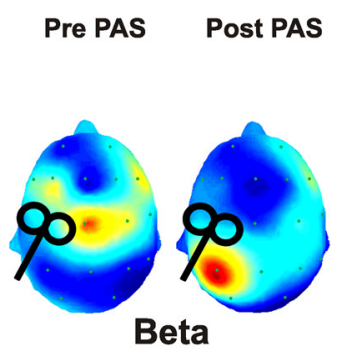

B

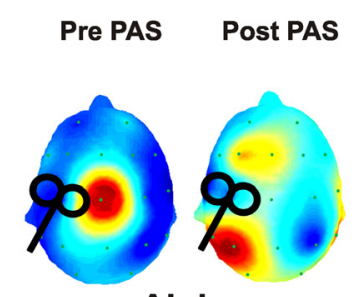

Alpha

C

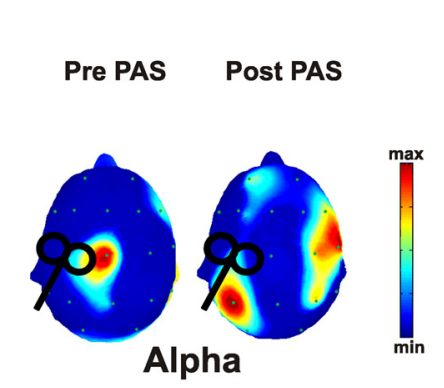

PPC-M1PA +5ms

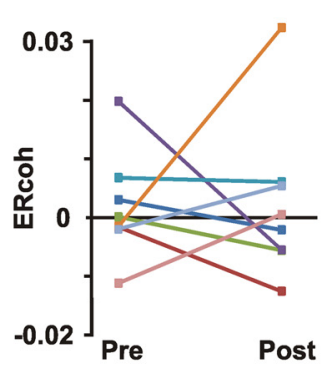

PPC-M1PA -5ms
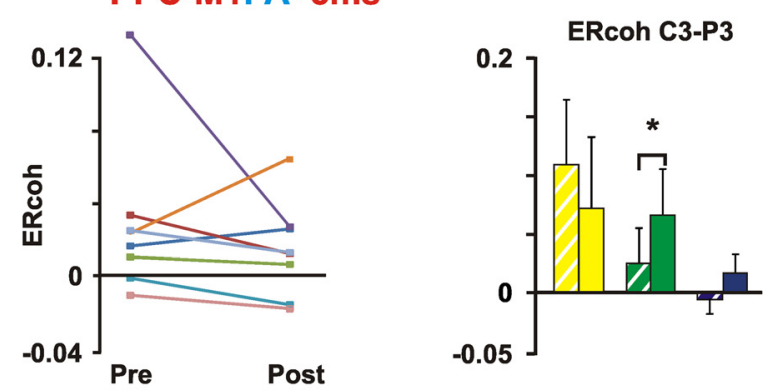

PPC-M1AP +5ms
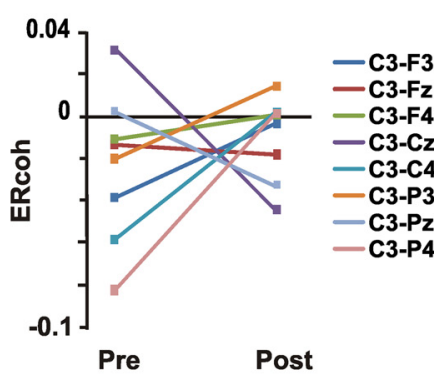

Figure 5. M1 event-related coherence changes induced by distinct PAS protocols. The scalp distribution maps on the right part of each panel show grand average of event-related coherence transformation of all electrodes referenced to (3 electrode (the closest electrode to M1 hot-spot), as a function of the experimental condition and Time (pre-PAS vs post-PAS) for the significantly modulated frequency band. Event-related coherence values are color coded according to the color bar. The middle part of each panel depicts ERcoh changes for a subsample of 9 electrodes surrounding the parietal end motor areas of both hemisphere for the significantly modulated frequency band. On the right for each panel, average data of the ERcoh for the C3-P3 pair before and after PAS stimulation are shown for all investigated frequencies (theta, alpha, and beta). Bars correspond to the SEM, asterisks mark significant differences ( $p<0.05$ ). $\boldsymbol{A}$, PPC-M1 $1_{\mathrm{PA}}+5 \mathrm{~ms}$ resulted in a decrease ERcoh between $C 3$ and central electrodes and a concurrent increase in coherence between $C 3$ and $P 3$. The change in ERcoh was significant for the beta band. $B$, PPC-M1 ${ }_{P A}-5 \mathrm{~ms}$ resulted in a decrease ERcoh between $C 3$ and $C Z$ and a concurrent increase in coherence between $C 3$ and P3. The change in ERcoh was significant for the alpha band. $C$, PPC-M $1_{A P}+5 \mathrm{~ms}$ resulted in a decrease ERcoh between $\mathrm{C} 3$ and $\mathrm{C}$ and a concurrent increase in coherence between C 3 and P3. The change in ERcoh was significant for the alpha band.

2009; Veniero et al., 2011). Despite that, ANOVA revealed that none of the PAS protocol was able to modulate theta, alpha, or beta power regardless of whether the single pulse TMS was used to test for $\mathrm{M} 1$ or PPC activity. In particular, after PPC-M1 $1_{\mathrm{PA}}$ PAS $-5 \mathrm{~ms}$ and PPC-M $1_{\mathrm{PA}}$ PAS - 5ms, ANOVA performed over the activity evoked by M1 stimulation revealed no main effect of Time nor a Time $\times$ Electrode interaction (all $p>0.05$ ). However, a significant main effect of Electrode was found when testing for alpha band modulations (PPC-M1PA PAS $-5 \mathrm{~ms}: F_{(19,209)}=$ 4,$23 ; p=0.000 ;$ PPC-M1 $1_{\mathrm{PA}}$ PAS $\left.-5 \mathrm{~ms}: F_{(19,209)}=4.61 ; p<0.01\right)$. These effects were due to significant higher values of alpha and beta power recorded from C3 and $\mathrm{Cz}$ (all $p<0.001$ ) compared with all other recording sites. No significant effect emerged from the analysis performed for the PPC-M $1_{\mathrm{AP}} \mathrm{PAS}-5 \mathrm{~ms}$ condition. Similar results emerged for the analysis of PPC stimulation. In- deed, a significant main effect of Electrode also emerged for the alpha band activity. Post hoc tests revealed that central electrodes $(\mathrm{C} 3, \mathrm{Cz}$, and C4) were indeed characterized by higher values of alpha-evoked activity (all $p<0.05$ ).

\section{Interregional functional connectivity}

When considering M1 stimulation (Fig. 5), ANOVA performed on interregional functional connectivity (ERcoh) values revealed that PPC-M1 $1_{\mathrm{PA}} \mathrm{PAS}-5 \mathrm{~ms}$ induced a significant modulation of functional beta band connectivity. Indeed, a significant Time $\times$ Pair interaction $\left(F_{(18,198)}=2.36 ; p=0.009\right)$ indicated an increase in functional coupling between P3 and C3 electrodes ( $p=0.005)$. No significant effects were found neither for other electrode pairs, nor for alpha and theta band coherence (all $p>0.05$ ). $\mathrm{PPC}-\mathrm{M} 1_{\mathrm{PA}} \mathrm{PAS}-5 \mathrm{~ms}$ also caused a change in coherence, but this 
A

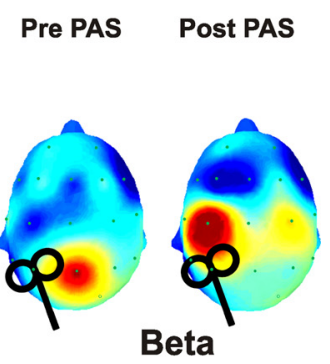

B

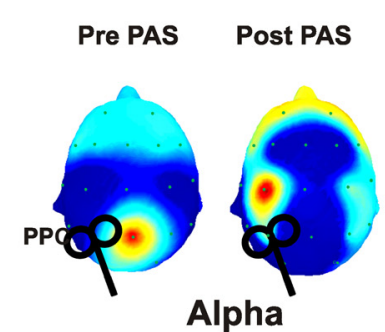

C

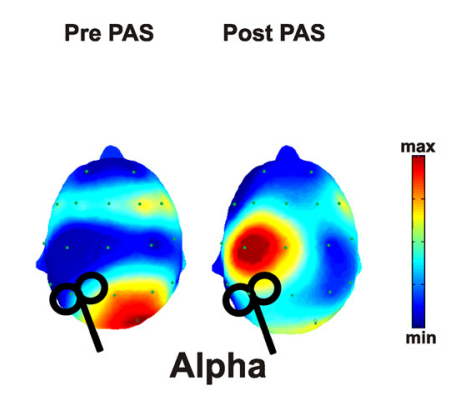

PPC-M1PA +5ms

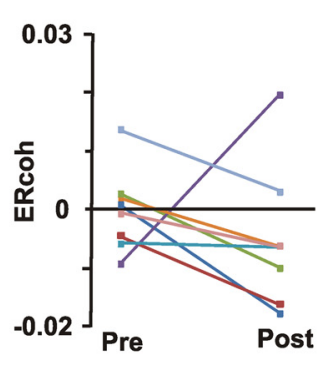

PPC-M1PA -5ms

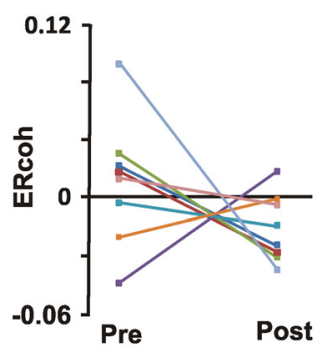

PPC-M1AP +5ms

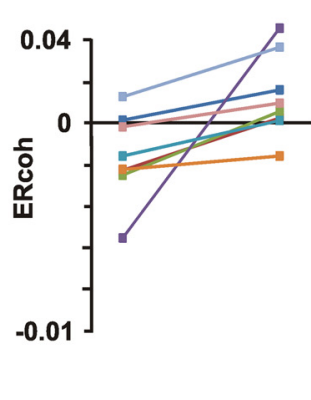

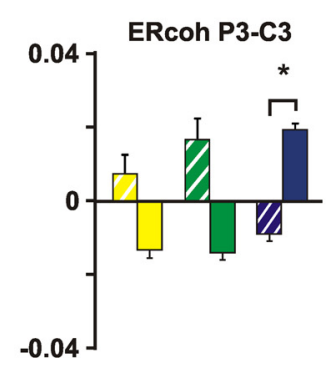

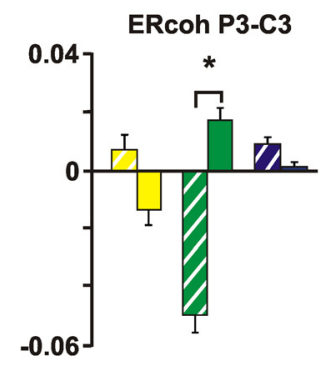

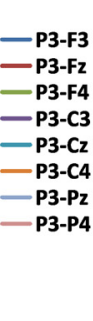

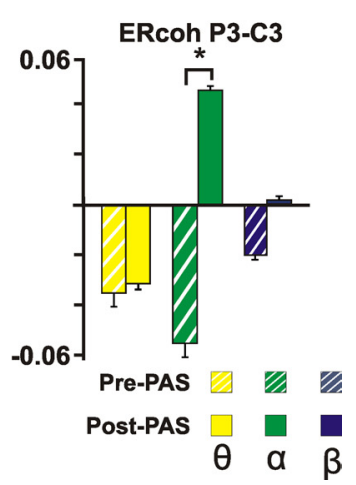

Figure 6. PPC event-related coherence changes induced by distinct PAS protocols. The scalp distribution maps on the right part of each panel show grand average of event-related coherence transformation of all electrodes referenced to the P3 electrode (the closest electrode to PPC hot-spot), as a function of the experimental condition and Time (pre-PAS vs post-PAS) for the significantly modulated frequency band. Event-related coherence values are color coded according to the color bar. The middle part of each panel depicts ERcoh changes for a subsample of 9 electrodes surrounding the parietal end motor areas of both hemisphere for the significantly modulated frequency band. On the right for each panel, average data of the ERcoh for the C3-P3 pair before and after PAS stimulation are shown for all investigated frequencies (theta, alpha, and beta). Bars correspond to the SEM, asterisks mark significant differences $(p<0.05) . A_{1}$ PPC-M1 $1_{P A}+5 \mathrm{~ms} \mathrm{resulted}$ in a decrease ERcoh between $C 3$ and central electrodes and a concurrent increase in coherence between $C 3$ and P3. The change in ERcoh was significant for the beta band. $B$, PPC-M1 1 PA $-5 \mathrm{~ms}$ resulted in a decreased ERcoh between $C 3$ and $C z$ and a concurrent increase in coherence between $C 3$ and P3. The change in ERcoh was significant for the alpha band. C, PPC-M1 $1_{A P}+5 \mathrm{~ms}$ resulted in a decreased ERcoh between $\mathrm{C} 3$ and $\mathrm{Z}$ and a concurrent increase in coherence between C 3 and P3. The change in ERcoh was significant for the alpha band.

condition modulated the alpha band ERcoh, as revealed by a significant Time $\times$ Pair interaction $\left(F_{(18,198)}=1.73 ; p=0.03\right)$. $\mathrm{PPC}-\mathrm{M} 1_{\mathrm{PA}} \mathrm{PAS}-5 \mathrm{~ms}$ led to an increase in coupling between $\mathrm{C} 3$ and P3 $(p=0.003)$ and to a concurrent decrease of ERcoh between $\mathrm{C} 3-\mathrm{Cz}(p=0.02)$. No significant effects were found neither for other electrode pairs, nor for beta and theta frequency (all $p>0.05)$. When comparing ERcoh values of before and after $\mathrm{PPC}-\mathrm{M} 1_{\mathrm{AP}}$ PAS $-5 \mathrm{~ms}$, a significant Time $\times$ Pair interaction $\left(F_{(18,126)}=2.65 ; p=0.017\right)$ emerged again for the alpha band. Post hoc tests indicated an increased coupling between C3 and P3 electrodes $(p=0.01)$ and a concurrent decreased ERcoh between the $\mathrm{C} 3$ and $\mathrm{Cz}$ pair $(p=0.02)$. No significant effects were found neither for other electrode pairs nor frequency.

When PPC connectivity was considered by calculating ERcoh during parietal stimulation (Fig. 6), ANOVA confirmed the re- sults found for M1 ERcoh modulation. Accordingly, PPC-M1 $1_{\mathrm{PA}}$ PAS $-5 \mathrm{~ms}$ induced a significant modulation of functional connectivity in the beta band, as shown by a Time $\times$ Pair interaction $\left(F_{(18,198)}=2,44 ; p=0.001\right)$ due to an increased coherence between P3 and C3 ( $p=0.0001)$ after PAS. No additional modulations were found for the theta and alpha band or for the other electrodes pairs (all $p>0.05$ ). Alpha ERcoh was significantly modulated by $\mathrm{PPC}-\mathrm{M} 1_{\mathrm{PA}} \mathrm{PAS}-5 \mathrm{~ms}$ protocol. A significant Time $\times$ Pair interaction $\left(F_{(18,198)}=4,43 ; p=0.001\right)$ was indeed found. Post hoc revealed an increased alpha band coupling for the P3-C3 pair $(p=0.029)$ and a concurrent decrease of coupling for the $\mathrm{P} 3-\mathrm{Pz}$ pair $(p=0.003)$. No significant effect over other frequency bands or electrodes pairs emerged (all $p>0.05$ ). Finally, PPC stimulation performed after PPC- $\mathrm{M} 1_{\mathrm{AP}}$ PAS $-5 \mathrm{~ms}$ revealed a significant modulation of alpha activity coherence after PAS 
(Time $\times$ Pair: $\left.F_{(18,126)}=1,81 ; p=0.03\right)$. The modulation resulted in an increased alpha coupling between the $\mathrm{C} 3$ and $\mathrm{P} 3$ pair $(p=0.001)$. Again, no significant effects over other frequency bands or electrodes pairs emerged (all $p>0.05$ ).

No significant correlation emerged between TEP amplitude modulations and increased coherence between C3 and P3.

\section{Discussion}

The current TMS-EEG approach allowed us to explore the cortical changes caused by corticocortical PAS. Our main result was that all protocols enforced the connectivity between the two areas and that the LTP- or LTD-like effects in M1 were associated with an increased communication in different oscillatory activity, suggesting that the connectivity in the alpha and beta bands could have distinct functional implications.

When looking at TEPs at baseline, TMS pulse elicited several responses with latency and scalp distributions matching those already described (Komssi et al., 2002; Bonato et al., 2006; Litvak et al., 2007). However, the activity evoked by PPC stimulation was smaller in amplitude than TEPs evoked by M1 stimulation. The topographical distribution indicated a more dynamic pattern after M1 stimulation, characterized by a clearer spread of activation toward connected areas, including nonstimulated contralateral hemisphere. The difference between M1 and PPC response further demonstrates that responsiveness to TMS could be different across distinct cortical areas because of several factors, such as a variable distance from the scalp to the cortex, a different anatomical organization, and anatomical connections (Kähkönen et al., 2004). GMFP amplitude revealed that the repetitive activation of the two areas was unable to modulate PPC activity, whereas all experimental manipulations led to changes in the $\mathrm{M} 1$ reactivity. In particular, when PAS increased corticospinal excitability (PPC-M1 $1_{\mathrm{PA}}$ PAS $-5 \mathrm{~ms}$, PPC-M1 $1_{\mathrm{AP}} \mathrm{PAS}+$ $5 \mathrm{~ms}$ ), the activity evoked within $18 \mathrm{~ms}$ after the pulse decreased in amplitude, whereas an increased cortical response (PPC-M1 $1_{\mathrm{PA}}$ PAS - 5ms) was associated with a decrease of MEP amplitude. Given the topographical distribution and source reconstruction performed in previous studies (Litvak et al., 2007), we hypothesize that this activity could represent an inhibitory process initiated by the stimulated motor areas. However, the relationship between TEP and MEP amplitude is far from being understood. Previous studies found a direct correlation between MEP and TEP amplitude (Esser et al., 2006; Mäki and Ilmoniemi, 2010). As an example, Esser et al. (2006) found that high-frequency repetitive TMS, which induces LTP-like phenomena, concurrently generates MEP and TEP amplitude increase. This discrepancy could be due to the different applied protocols. Indeed, rTMS was exclusively applied to M1 (Esser et al., 2006), whereas in the present study the plasticity-like effects were generated by a repeated activation of two interconnected areas. Alternatively, given the weak correlations found by several studies (Lepage et al., 2008; Mäki and Ilmoniemi, 2010), EEG indexes could only be limited predictors of MEPs size, likely because EEG signals typically reflect the activity in a large cortical region, while cortical activity related to MEPs should be specific to the neurons controlling the target muscles and may be influenced by spinal excitability.

Two protocols (PPC-M1 $1_{\mathrm{PA}} \mathrm{PAS}-5 \mathrm{~ms}, \mathrm{PPC}-\mathrm{M} 1_{\mathrm{AP}} \mathrm{PAS}+$ $5 \mathrm{~ms}$ ) were also associated with a modulation of a late GMFP component (P4). This activity showed a smaller amplitude if compared with the $\mathrm{P} 1$, but showed a comparable topographical distribution and a parallel modulation, likely further indicating a local change in inhibitory response, restricted to the M1 activity.
Several reasons could account for the lack of PAS effects over PPC TEPs. A simple explanation would be that during PAS, the subthreshold TMS intensity (90\% RMT) was not sufficient to induce plastic changes over PPC, even when the PPC followed M1 stimulation. Moreover, during the pretesting and posttesting phase, TMS intensity was adjusted to the value needed to consistently activate M1 ( 130\%RMT), but despite this higher intensity the TEPs were smaller than those found in M1. Therefore, it seems that the PPC is globally less responsive to TMS than M1. Aside from these considerations, the present results fit well with our previous results (Koch et al., 2013) in that they support the hypothesis that PPC could be regarded as presynaptic target, not modulated by the paired stimulation, and of M1 as the postsynaptic target.

When looking at the power values, in line with previous studies (Rosanova et al., 2009; Veniero et al., 2011), the stimulation performed over M1 mainly induced an alpha activity, whereas parietal stimulation consistently generated beta band activity. However, we found no effects on power values after PAS administration.

The main findings of the present study is that the local dynamics of M1 revealed by MEP and TEP amplitude modulations are associated with remarkable changes in the cross talk between the cortical areas, which are transmitted by specific cortical rhythms. For both hebbian and anti-hebbian STPD the connectivity increases selectively between the stimulated cortical areas. Moreover, a sharp increase in the alpha band was found when MEP modulation indexed an LTP-like effect and in the beta band when MEPs modulation suggested an LTD-like effect. These results therefore suggest that the repetitive association of parietal and motor activation enforce the communication between the two areas. The opposite protocol outcome found for MEP amplitude is not associated with coherence changes of opposite sign (increase vs decrease) but with an increased coupling in distinct brain rhythms, thus likely suggesting that distinct oscillatory activity carries different information. Coherence of oscillatory brain activity has been indicated as an important neurophysiological mechanism providing the basis for cell assembly cooperation that can communicate through the synchronization at specific frequencies. It has been proposed that only coherently oscillating neuronal groups can effectively interact, because their communication windows for input and output are open at the same time (Fries, 2005). Thus, an increase in coherence seems to represent a natural candidate to bind together cell assemblies that need to communicate for the associative learning to take place (Miltner et al., 1999; Knoblauch et al., 2012). Interestingly, several recent studies showed a correlation between alpha band connectivity and LTP-like phenomena induction. Freyer et al. (2012) also showed that other hebbian-like learning processes such as a repetitive tactile stimulation results in an improved tactile acuity strongly associated with an increased alpha band connectivity. In addition to these considerations, the alpha and beta involvement in our associative protocol could speculatively be related to the brain rhythms characterizing the motor and parietal cortex. Indeed, it is well known that alpha and beta bands reflect typical rolandic rhythms (Salmelin and Hari, 1994), and more recently Rosanova et al. (2009) demonstrated that in resting condition PPC naturally oscillate at beta frequency. Therefore, the manipulation of parietomotor connections could lead to a change in the PPC-M1 communication in the rhythms already characterizing their activity. Since there was no alpha or beta power differences between the pre-PAS and post-PAS testing in all experimental conditions, the increase of parietomotor coherence can only be 
interpreted as an increase in phase coupling between these regions (Sauseng et al., 2005). The repeated pairing of activation therefore led to an increase in the efficacy of the communication between the two areas without requiring a concurrent increase of the signal strength.

Previous works have indicated that PPC and M1 are highly interconnected areas, but mainly through an indirect pathway involving the premotor area and by weak direct PPC-M1 pathways (Makris et al., 2005; Koch et al., 2010). Therefore, the increase of coherence could be explained by a strengthening of these recurrent connections. However, as recently proposed (Sherman and Guillery, 2011), all cortical areas having a corticocortical connection also have parallel trans-thalamic connections. Accordingly, when the trans-thalamic link does not assist the corticocortical pathways the target activation could be too weak to generate a functional link. Consequently, we propose that the increased coupling between PPC and M1 could be explained by a coactivation of both corticocortical and transthalamic pathways, which basically increased the probability of the coincidence of presynaptic and postsynaptic activation (Sherman and Guillery, 2011), leading the cortical areas to be functionally linked.

Previous studies already demonstrated that M1 coherence can be manipulated by TMS protocol known to induce LTP- or LTDlike effects. Monofocal TMS applied at low frequency $(1 \mathrm{~Hz})$ has been associated with an increased alpha coherence between premotor and motor areas and a concurrent decreased MEP amplitude (Strens et al., 2002), whereas TMS applied at high frequency ( $5 \mathrm{~Hz}$ ) has been found to be related to alpha and beta band coherence decrease between motor and premotor or motor and parietal cortices and a concurrent increase in MEP amplitude (Oliviero et al., 2003; Fuggetta et al., 2008). However, these results cannot be directly linked to our data, since in the present study the connectivity was directly manipulated by means of bifocal TMS stimulation. To our knowledge only one study evaluated the effect of bifocal TMS over long-range connectivity (Plewnia et al., 2008) between occipital and motor areas. However, the study by Plewnia et al. (2008) was designed to induce an alpha increase in coherence by delivering trains of TMS pulses at alpha frequency over the two regions.

In conclusion, we demonstrate that antithetic forms of STDP are associated with distinct dynamics at cortical level. Plastic after-effects are associated with changes in M1 cell activity and most interestingly that the information is transmitted from one region to another by different cortical rhythms. The current data could be important because they demonstrate the possibility to selectively manipulate the functional connectivity between two cortical areas. Further studies linking these data to behavioral outcomes are needed to elucidate the functional significance of increasing the efficacy of communication in distinct oscillatory activity and the potential impact of these manipulations in promoting circuit reorganization in patients.

\section{References}

Arai N, Müller-Dahlhaus F, Murakami T, Bliem B, Lu MK, Ugawa Y, Ziemann U (2011) State-dependent and timing-dependent bidirectional associative plasticity in the human SMA-M1 network. J Neurosci 31:15376-15383. CrossRef Medline

Bonato C, Miniussi C, Rossini PM (2006) Transcranial magnetic stimulation and cortical evoked potentials: a TMS/EEG co-registration study. Clin Neurophysiol 117:1699-1707. CrossRef Medline

Buch ER, Johnen VM, Nelissen N, O'Shea J, Rushworth MF (2011) Noninvasive associative plasticity induction in a corticocortical pathway of the human brain. J Neurosci 31:17669-17679. CrossRef Medline
Day BL, Dressler D, Maertens de Noordhout A, Marsden CD, Nakashima K, Rothwell JC, Thompson PD (1989) Electric and magnetic stimulation of human motor cortex: surface EMG and single motor unit responses. J Physiol 412:449-473. Medline

Delorme A, Makeig S (2004) EEGLAB: an open source toolbox for analysis of single-trial EEG dynamics including independent component analysis. J Neurosci Methods 134:9-21. CrossRef Medline

Di Lazzaro V, Pilato F, Oliviero A, Dileone M, Saturno E, Mazzone P, Insola A, Profice P, Ranieri F, Capone F, Tonali PA, Rothwell JC (2006) Origin of facilitation of motor-evoked potentials after paired magnetic stimulation: direct recording of epidural activity in conscious humans. J Neurophysiol 96:1765-1771. CrossRef Medline

Esser SK, Huber R, Massimini M, Peterson MJ, Ferrarelli F, Tononi G (2006) A direct demonstration of cortical LTP in humans: a combined TMS/EEG study. Brain Res Bull 69:86-94. CrossRef Medline

Freyer F, Reinacher M, Nolte G, Dinse HR, Ritter P (2012) Repetitive tactile stimulation changes resting-state functional connectivity-implications for treatment of sensorimotor decline. Front Hum Neurosci 6:144. Medline

Fries P (2005) A mechanism for cognitive dynamics: neuronal communication through neuronal coherence. Trends Cogn Sci 9:474-480. CrossRef Medline

Froemke RC, Letzkus JJ, Kampa BM, Hang GB, Stuart GJ (2010) Dendritic synapse location and neocortical spike-timing-dependent plasticity. Front Synaptic Neurosci 2:29. Medline

Fuggetta G, Pavone EF, Fiaschi A, Manganotti P (2008) Acute modulation of cortical oscillatory activities during short trains of high-frequency repetitive transcranial magnetic stimulation of the human motor cortex: a combined EEG and TMS study. Hum Brain Mapp 29:1-13. CrossRef Medline

Ilmoniemi RJ, Virtanen J, Ruohonen J, Karhu J, Aronen HJ, Näätänen R, Katila T (1997) Neuronal responses to magnetic stimulation reveal cortical reactivity and connectivity. Neuroreport 8:3537-3540. CrossRef Medline

Kähkönen S, Wilenius J, Komssi S, Ilmoniemi RJ (2004) Distinct differences in cortical reactivity of motor and prefrontal cortices to magnetic stimulation. Clin Neurophysiol 115:583-588. CrossRef Medline

Katz LC, Shatz CJ (1996) Synaptic activity and the construction of cortical circuits. Science 274:1133-1138. CrossRef Medline

Knoblauch A, Hauser F, Gewaltig MO, Körner E, Palm G (2012) Does spike-timing-dependent synaptic plasticity couple or decouple neurons firing in synchrony? Front Comput Neurosci 6:55. Medline

Koch G, Fernandez Del Olmo M, Cheeran B, Ruge D, Schippling S, Caltagirone C, Rothwell JC (2007) Focal stimulation of the posterior parietal cortex increases the excitability of the ipsilateral motor cortex. J Neurosci 27:6815-6822. CrossRef Medline

Koch G, Oliveri M, Cheeran B, Ruge D, Lo Gerfo E, Salerno S, Torriero S, Marconi B, Mori F, Driver J, Rothwell JC, Caltagirone C (2008) Hyperexcitability of parietal-motor functional connections in the intact lefthemisphere of patients with neglect. Brain 131:3147-3155. CrossRef Medline

Koch G, Cercignani M, Pecchioli C, Versace V, Oliveri M, Caltagirone C, Rothwell J, Bozzali M (2010) In vivo definition of parieto-motor connections involved in planning of grasping movements. Neuroimage 51: 300-312. CrossRef Medline

Koch G, Cercignani M, Bonnì S, Giacobbe V, Bucchi G, Versace V, Caltagirone C, Bozzali M (2011) Asymmetry of parietal interhemispheric connections in humans. J Neurosci 31:8967-8975. CrossRef Medline

Koch G, Ponzo V, Di Lorenzo F, Caltagirone C, Veniero D (2013) Hebbian and anti-hebbian spike-timing-dependent plasticity of human corticocortical connections. J Neurosci 33:9725-9733. CrossRef Medline

Komssi S, Aronen HJ, Huttunen J, Kesäniemi M, Soinne L, Nikouline VV, Ollikainen M, Roine RO, Karhu J, Savolainen S, Ilmoniemi RJ (2002) Ipsi- and contralateral EEG reactions to transcranial magnetic stimulation. Clin Neurophysiol 113:175-184. CrossRef Medline

Lepage JF, Saint-Amour D, Théoret H (2008) EEG and neuronavigated single-pulse TMS in the study of the observation/execution matching system: are both techniques measuring the same process? J Neurosci Methods 175:17-24. CrossRef Medline

Letzkus JJ, Kampa BM, Stuart GJ (2006) Learning rules for spike timingdependent plasticity depend on dendritic synapse location. J Neurosci 26:10420-10429. CrossRef Medline 
Litvak V, Komssi S, Scherg M, Hoechstetter K, Classen J, Zaaroor M, Pratt H, Kahkonen S (2007) Artifact correction and source analysis of early electroencephalographic responses evoked by transcranial magnetic stimulation over primary motor cortex. Neuroimage 37:56-70. CrossRef Medline

Mäki H, Ilmoniemi RJ (2010) The relationship between peripheral and early cortical activation induced by transcranial magnetic stimulation. Neurosci Lett 478:24-28. CrossRef Medline

Makris N, Kennedy DN, McInerney S, Sorensen AG, Wang R, Caviness VS Jr, Pandya DN (2005) Segmentation of subcomponents within the superior longitudinal fascicle in humans: a quantitative, in vivo, DT-MRI study. Cereb Cortex 15:854-869. CrossRef Medline

Miltner WH, Braun C, Arnold M, Witte H, Taub E (1999) Coherence of gamma-band EEG activity as a basis for associative learning. Nature 397: 434-436. CrossRef Medline

Miniussi C, Thut G (2010) Combining TMS and EEG offers new prospects in cognitive neuroscience. Brain Topogr 22:249-256. CrossRef Medline

Mutanen T, Mäki H, Ilmoniemi RJ (2013) The effect of stimulus parameters on TMS-EEG muscle artifacts. Brain Stimul 6:371-376. CrossRef Medline

Ni Z, Charab S, Gunraj C, Nelson AJ, Udupa K, Yeh IJ, Chen R (2011) Transcranial magnetic stimulation in different current directions activates separate cortical circuits. J Neurophysiol 105:749-756. CrossRef Medline

Oliviero A, Strens LH, Di Lazzaro V, Tonali PA, Brown P (2003) Persistent effects of high frequency repetitive TMS on the coupling between motor areas in the human. Exp Brain Res 149:107-113. Medline

Pfurtscheller G, Lopes da Silva FH (1999) Event-related EEG/MEG synchronization and desynchronization: basic principles. Clin Neurophysiol 110:1842-1857. CrossRef Medline

Plewnia C, Rilk AJ, Soekadar SR, Arfeller C, Huber HS, Sauseng P, Hummel F, Gerloff C (2008) Enhancement of long-range EEG coherence by synchronous bifocal transcranial magnetic stimulation. Eur J Neurosci 27: 1577-1583. CrossRef Medline

Rizzo V, Siebner HS, Morgante F, Mastroeni C, Girlanda P, Quartarone A (2009) Paired associative stimulation of left and right human motor cortex shapes interhemispheric motor inhibition based on a Hebbian mechanism. Cereb Cortex 19:907-915. Medline

Rosanova M, Casali A, Bellina V, Resta F, Mariotti M, Massimini M (2009) Natural frequencies of human corticothalamic circuits. J Neurosci 29: 7679-7685. CrossRef Medline
Rossi S, Hallett M, Rossini PM, Pascual-Leone A; Safety of TMS Consensus Goup (2009) Safety, ethical considerations, and application guidelines for the use of transcranial magnetic stimulation in clinical practice and research. Clin Neurophysiol 120:2008-2039. CrossRef Medline

Rossini PM, Barker AT, Berardelli A, Caramia MD, Caruso G, Cracco RQ, Dimitrijević MR, Hallett M, Katayama Y, Lücking $\mathrm{CH}$, Maertens de Noordhout AL, Marsden CD, Murray NMF, Rothwell JC, Swash M, Tomberg C (1994) Non-invasive electrical and magnetic stimulation of the brain, spinal cord and roots: basic principles and procedures for routine clinical application. Report of an IFCN committee. Electroencephalogr Clin Neurophysiol 91:79-92. CrossRef Medline

Salmelin R, Hari R (1994) Characterization of spontaneous MEG rhythms in healthy adults. Electroencephalography and clinical neurophysiology 91:237-248. CrossRef Medline

Sauseng P, Klimesch W, Schabus M, Doppelmayr M (2005) Fronto-parietal EEG coherence in theta and upper alpha reflect central executive functions of working memory. Int J Psychophysiol 57:97-103. CrossRef Medline

Sherman SM, Guillery RW (2011) Distinct functions for direct and transthalamic corticocortical connections. J Neurophysiol 106:1068-1077. CrossRef Medline

Sjöström PJ, Rancz EA, Roth A, Häusser M (2008) Dendritic excitability and synaptic plasticity. Physiol Rev 88:769-840. CrossRef Medline

Strens LH, Oliviero A, Bloem BR, Gerschlager W, Rothwell JC, Brown P (2002) The effects of subthreshold $1 \mathrm{~Hz}$ repetitive TMS on cortico-cortical and interhemispheric coherence. Clin Neurophysiol 113:1279-1285. CrossRef Medline

Thut G, Veniero D, Romei V, Miniussi C, Schyns P, Gross J (2011) Rhythmic TMS causes local entrainment of natural oscillatory signatures. Curr Biol 21:1176-1185. CrossRef Medline

Veniero D, Bortoletto M, Miniussi C (2009) TMS-EEG co-registration: on TMS-induced artifact. Clin Neurophysiol 120:1392-1399. CrossRef Medline

Veniero D, Maioli C, Miniussi C (2010) Potentiation of short-latency cortical responses by high-frequency repetitive transcranial magnetic stimulation. J Neurophysiol 104:1578-1588. CrossRef Medline

Veniero D, Brignani D, Thut G, Miniussi C (2011) Alpha-generation as basic response-signature to transcranial magnetic stimulation (TMS) targeting the human resting motor cortex: a TMS/EEG coregistration study. Psychophysiology 48:1381-1389. CrossRef Medline 